\title{
BMJ Open Tuina for spasticity of poststroke: protocol of a systematic review and meta-analysis
}

\author{
Qiongshuai Zhang (D) , ${ }^{1}$ Guangcheng $\mathrm{Ji}^{2}{ }^{2}$ Fang Cao, ${ }^{3}$ Yihan Sun, ${ }^{4}$ Guanyu Hu, ${ }^{1}$ \\ Shaoqian Sun, ${ }^{5}$ Yanze Liu, ${ }^{1}$ Jiazhen Cao, ${ }^{1}$ Yufeng Wang, ${ }^{6}$ Xiaohong $\mathrm{Xu},{ }^{7}$ \\ Bailin Song ${ }^{1}$
}

To cite: Zhang Q, Ji G, Cao F, et al. Tuina for spasticity of poststroke: protocol of a systematic review and meta-analysis. BMJ Open 2020;10:e038705. doi:10.1136/ bmjopen-2020-038705

- Prepublication history for this paper is available online. To view these files, please visit the journal online (http://dx.doi org/10.1136/bmjopen-2020038705).

Received 23 March 2020 Revised 23 September 2020 Accepted 24 October 2020
Check for updates

(C) Author(s) (or their employer(s)) 2020. Re-use permitted under CC BY-NC. No commercial re-use. See rights and permissions. Published by BMJ.

For numbered affiliations see end of article.

Correspondence to Professor Bailin Song; jlsongb|@126.com

\section{ABSTRACT}

Introduction Spasticity is a common complication of poststroke, tuina is a widely used rehabilitation treatment, although there is a lack of supportive evidence on efficacy and safety for patients with poststroke spasticity. The aim of this systematic review is to assess and synthesis evidence of efficacy and safety of tuina for spasticity of poststroke.

Methods and analysis A comprehensive electronic search of EMBASE, MEDLINE, Cochrane Library, Web of Science, Wiley, Springer, PEDro, Chinese Science Citation Database, China National Knowledge Infrastructure, Chinese Biomedical Literature Database, Chinese Scientific and Journal Database (VIP), Wanfang Database (Wanfang), Japanese medical database (CiNii), Korean Robotics Institute Summer Scholars and Thailand Thai-Journal Citation Index Centre will be conducted to search literatures of randomised controlled trials of tuina for spasticity of poststroke survivors range from the establishment to 1 January 2020.

There is no time of publication limitations. The primary outcome will be measured with the Modified Ashworth Scale, and the second outcome will include Fugl-Meyer Assessment Scale, surface electromyogram RMS value, the Modified Barthel Index, Stroke Specific Quality of Life Scale, quality of life 36-Item Short-Form Health Survey and Visual Analogue Scale. Cochrane Handbook for Systematic Reviews of Interventions will be used to assess the risk of bias, and GRADE will be used to access the confidence in cumulative evidence. The protocol will be conducted according to approach and Preferred Reporting Items for Systematic Review and Meta-Analysis Protocols 2015. Ethics and dissemination Ethical approval will not be required, for no primary data of individual patients were collected. We will publish the findings in a peer-reviewed journal.

PROSPERO registration number CRD42020163384.

Stroke has been the first risk factor of death in China. ${ }^{1}$ It is also one of the diseases with high mortality and disability rate in the world. ${ }^{2}$ Limb spasticity is a common complication of poststroke patients. ${ }^{34}$ Recent study shows that about $17 \%-43 \%$ of stroke patients had limb spasticity, ${ }^{5-7}$ and the medical cost of patients with poststroke limb spasticity is about four
Strengths and limitations of this study

- This is the first comprehensive systematic review focused on efficacy and safety of tuina for spasticity of poststroke.

- Only randomised controlled trials (RCTs) (not included quasi-RCTs) will be included in this study.

- We searched databases of English, Chinese, Japanese, Korean and Thailand, while other languages may be ignored.

times as much as poststroke patients without spasticity. ${ }^{89}$ Limb spasticity not only severely restricts the ability of patients, reduces the quality of life, but also causes psychological impact on patients' rehabilitation, and brings a great burden to families and society. ${ }^{10-14}$

Physical therapy, oral or injection drug therapy and operation therapy are commonly used in Western medicine to treat poststroke spasticity at present. Oral drugs such as baclofen, eperisone, hydrochloride and diazepine have large side effects which hinder the recovery of motor function with long time taking. ${ }^{15}$ Botulinum toxin treatment is difficult to achieve long-term results, and it is often injected for moderate or severe cases of poststroke spasticity. ${ }^{1617}$ Physical therapy often requires active exercise coordination of patients; however, patients with severe conditions are often unable to cooperate. Surgical treatment is traumatic and a large number of patients often find it difficult to accept. At present, much more of the patients with spasticity after stroke choose external treatment. In China, many external treatment methods of traditional Chinese medicine (TCM) are applied to the treat this disease.

Tuina is an ancient form of external treatment method, which was based on the meridian and acupoint theory of TCM, and uses specific operation skill acting on the surface or acupoints of the patient's body to 
treat diseases. ${ }^{18} 19$ Tuina has been widely used in China for hundreds of years and increasingly practiced in Western countries in recent years. Systematic evaluation ${ }^{20}$ shows that acupuncture is efficacy and safety in the treatment of limb spasticity after stroke. Acupuncture and tuina belong to the external treatment of TCM, and both are based on the same theory of meridians and acupoints. However, it is still unclear whether the effectiveness of acupuncture is also applicable to tuina in the treatment of poststroke spasticity. We want to know the effect of tuina, which has the characteristics of intervention and low cost, for poststroke spasticity. At present, there is no systematic review of tuina in the treatment of poststroke limb spasticity, so this study will evaluate the efficacy and safety of tuina in the treatment of poststroke limb spasticity, and provide evidence for clinical decision-making of massage.

\section{METHODS}

The systematic review will be performed following the guideline of Preferred Reporting Items for Systematic Review and Meta-Analysis Protocols (PRISMA-P) $2015 .{ }^{21}$

\section{Inclusion criteria}

Types of studies

We will include randomised controlled trials (RCTs) (not included quasi-RCTs) of tuina for poststroke spasticity in the treatment groups. If multiarm RCTs comes, we will select the group which used tuina and another without tuina for analysis. We will select the first stage of cross over RCTs, in which tuina was first used in one group. RCTs' language of English, Chinese, Japanese, Korean and Thai will be included.

\section{Types of participants}

Patients suffering postacute phase of post-stroke spasticity ( $>18$ yearsold) will be included. Stroke (cerebral infarction or cerebral haemorrhage) is diagnosed according to WHO criteria, ${ }^{22}$ participants have the symptoms of limb muscle tension increase, and the Modified Ashworth Scale (MAS) score is grade 1-2. Participants of any sex and ethnicity will be enrolled.

\section{Types of interventions}

The treatment group using tuina while the control group receives treatment of oral medication, acupuncture, Chinese herbal medication, physical therapy, surgery, botox injections and so on or even with no treatment will be included.

\section{Types of outcome measures}

\section{Primary outcome}

Muscle tone will be evaluated by the MAS. MAS is a clinical instrument which is commonly used for measuring spasticity, and studies have proofed its reliability. ${ }^{23-25}$

\section{Secondary outcome}

Motor function was assessed with Fugl-Meyer Assessment Scale (FMA) or Simplified FMA Scale.
Muscle strength will be defined by surface electromyogram root mean square value (RMS).

Activities of daily living will be assessed by the Modified Barthel Index.

Quality of life will be measured by Stroke Specific Quality of Life Scale or quality of life 36-Item Short-Form Health Survey (SF-36).

Limb pain will be assessed by Visual Analogue Scale.

\section{Safety outcome}

Skin abrasions.

\section{Exclusion criteria}

- Repeatedly published studies.

- Experiences, letters, systematic reviews and animal experiments.

- Tuina was not only in the experimental group but also in the control group.

- Articles without full text or with data which are missed or cannot be used.

\section{Search strategy}

Electronic searches

The published electronic literature will be searched in EMBASE, MEDLINE (by PubMed), Cochrane Library, Web of Science, Wiley, Springer, PEDro, Chinese Science Citation Database, China National Knowledge Infrastructure, Chinese Biomedical Literature Database, Chinese Scientific and Journal Database (VIP), Wanfang Database, Japanese medical database (CiNii), Korean Robotics Institute Summer Scholars and Thailand Thai-Journal Citation Index Centre. We will also cheek reference lists, and the literature will be searched range from the establishment to 1 January 2020.

The search strategy is developed according to published reviews. ${ }^{26} 27$ The detail search strategy of MEDLINE (by PubMed) is listed in table 1, while the search strategy will be modified according to other different databases.

\section{Data collection and analysis \\ Selection of literature}

Two authors (YL, JC) will identify studies according to the inclusion criteria independently. First, they will eliminate duplicate researches by using EndNote software (V.x9.0). Second, screening the title and abstract, if necessary, reading the full article to confirm if it should be included. They also use EndNote software to manage the included studies. The screening operation is performed as shown in figure 1. If there is disagreement during the screening process, discuss with the third experts (GJ) to make a decision.

\section{Data extraction and management}

Two authors (SS and YW) will extract data from the included studies independently. In multiarm RCTs, we will extract data from RCTs of two arms, while we will select one group which contains the treatment of tuina as the treatment group, we will also choose another group the treatment of which without tuina as the control group. 
Table 1 MEDLINE (by PubMed) search strategy

Spasticity of \#1.Stroke[MeSH] OR Apoplexy [Tiab] OR post stroke post-stroke[tiab] OR poststroke[tiab] OR Apoplectic [Tiab] OR Apoplexia [Tiab] OR Cerebral hemorrhage [Tiab] OR Ich [Tiab] OR Cerebrovascular accident [Tiab] OR Cerebrovascular disorders [Tiab] OR Cerebral embolism [Tiab] OR Brain embolism [Tiab] OR Embolic stroke [Tiab] OR Cerebral infarct OR $\mathrm{cva}^{*}[\mathrm{tiab}]$

\#2.spasm[Mesh] OR dystonia[tiab] OR paraparesis,spastic[tiab] OR muscle spasticity $^{\star}[$ tiab] OR muscle hypertonia [tiab] OR muscle rigidity ${ }^{\star}[$ tiab] OR muscle tonus[tiab] OR spas*[tiab] OR high tone[tiab]

\#3. \#1 AND \#2

$\begin{array}{ll}\text { Tuina } & \text { \#4.Tuina[tiab] or Massage[tiab] or } \\ & \text { Acupressure[tiab] or Rub[tiab] or } \\ & \text { Massageing[tiab] or Massotheraty[tiab] or } \\ & \text { manipulation[tiab] }\end{array}$

Randomised \#5. Randomised controlled trial [pt] OR controlled controlled clinical trial [pt] OR randomized trial [tiab] OR placebo [tiab] OR drug therapy [sh] OR randomly [tiab] OR trial [tiab] OR groups [tiab)

Final search 6.\#3 AND \#4 AND \#5 strategy

The general information consists of title, publication year, authors, country, language and journal source; information of participants: gender, age, stroke type (cerebral infarction or cerebral haemorrhage), duration of onset and sample size; information of intervention characteristics: type, session, duration and follow-up time and outcome information about primary outcome, second outcome, observation time points and adverse effects.

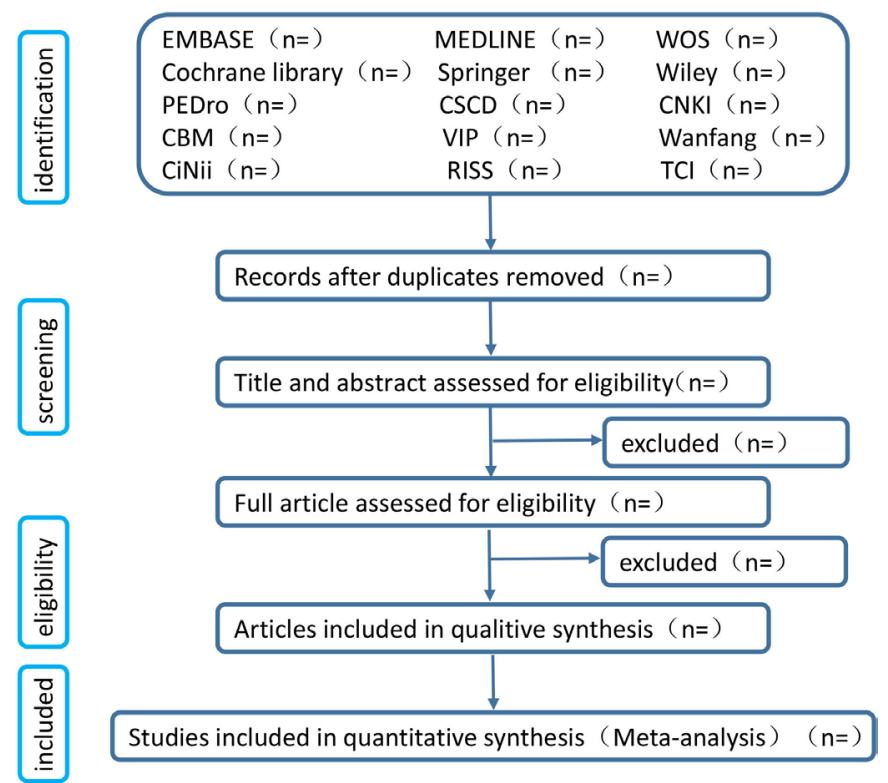

Figure 1 The screening process.

\section{Assessment of risk of bias in included studies}

Two independent authors (QZ and FC) will evaluate the risk of bias by using the Cochrane Collaboration bias risk assessment tool to assess the risk bias of the literature included in the systematic review. The two authors will assess the risk of bias of sequence generation, allocation concealment, blinding of participants personnel and outcome assessment, incomplete outcome data, selective outcome reporting and other bias. The evaluation grades are low, high and unclear risk of bias.

\section{Measures of treatment effect}

Two independent authors (YS and QZ) will use the mean difference (MD) or standard MD with $95 \%$ CI for continuous data of final measurements, the other binary data will be changed into relative risk (RR) form.

\section{Dealing with missing data}

When the included article lacks some important information, we will try to contact the correspondence author through e-mail, phone or other contacts. If we can't get the information through the ways above, we will turn to the following strategies to evaluate the potential influence of missing data: ${ }^{28}$

- Worst-case scenario analysis: all participants with missing data counted as failures.

- Extreme worst-case/best-case scenario analysis: participants with missing outcome data in the exercise arm counted as failures and in the control arm as success and vice versa.

\section{Assessment of heterogeneity}

We will use Q-test and $\mathrm{I}^{2}$ statistic to assess the heterogeneity of the included studies, as the criteria: $\mathrm{I}^{2}<50 \%$ indicates low heterogeneity, while $\mathrm{I}^{2}>50 \%$ indicates high heterogeneity,

\section{Assessment of reporting bias}

We will construct funnel plots to assess asymmetry, only if at least 10 RCTs are included.

\section{Data synthesis}

The meta-analysis of intervention and outcome measures methods will be conducted by RevMan V.5.3.5 software (the Cochrane Collaboration, Oxford, England). If the statistical heterogeneity is low $\left(\mathrm{P}>0.1\right.$, or $\left.\mathrm{I}^{2}<50 \%\right)$, we will use the fixed-effect model to combine the data, while if the statistical heterogeneity is high $\left(\mathrm{P}<0.1\right.$, or $\left.\mathrm{I}^{2}>50 \%\right)$, we will use the random-effect model. However, if the heterogeneity level much significant, a descriptive analysis will be performed.

\section{Subgroup analysis and investigation of heterogeneity}

We will perform subgroup analysis to assess heterogeneity of the study according to the following potential factors from the available sufficient data:

- Age.

- Sex. 
- Different types of stroke (Cerebral haemorrhage or cerebral infarction).

- Different types of tuina.

- Different time/course of treatment.

- Different parts of affected limbs (upper limb or lower limb).

- Different types of control group (acupuncture, placebo, oral/injection drug or no treatment).

We may make meta-regressions according to age and the different time/course of treatment if heterogeneity is obvious.

\section{Sensitivity analysis}

We will perform the sensitivity analysis to evaluate the robustness and reliability of the pooled results. If the results are not stable, we may turn to removing studies of high risk of bias, or cheek up processing method of missing data (worst-case scenario analysis: all participants with missing data counted as failures; extreme worstcase/best-case scenario analysis: participants with missing outcome data).

\section{Grading of evidence quality}

We will use the Grading of Recommendations Assessment, Development and Evaluation ${ }^{29}$ to access the confidence in cumulative evidence. Risk of bias, heterogeneity, indirectness, imprecision and publication bias will be assessed, and the results will be divided into four levels: high, moderate, low and very low.

\section{Amendments}

We will show all the amendments with detailed description and rationale in the amendments of this study.

\section{Ethics and dissemination}

There is no need of ethical approval in this study, because there is nothing of the data which has a relationship with an individual patient. We will complete this systematic review according to the PRISMA guidelines. The review will provide an assessment of effect and safety of tuina for spasticity of poststroke. We will publish the findings in a peer-reviewed, open assess journal and the finished systematic review and meta-analysis will be disseminated online, which would be obtained freely for anyone. The results may contribute to improving the therapeutic strategy of patients with poststroke spasticity.

\section{Patient and public involvement}

No patient or public will be involved in our study directly. We only use data that existed in studies published.

\section{DISCUSSION}

This systematic review will focus on the efficacy and safety of tuina for spasticity of poststroke. Tuina is a traditional Chinese physical therapy, which is effective for 516 diseases in China, ${ }^{30}$ of which spasticity is included. Clinical reports show tuina is well in treatment of spasticity of poststroke; however, high-quality study still did not appear. We conducted this review with the aim to provide better evidence and guide for clinical decision-making. We plan to publish this review within 1 year since the protocol published, then we will update it every 3 years.

\section{Author affiliations}

${ }^{1}$ Acupuncture and Tuina Department, Changchun University of Chinese Medicine, Changchun, China

${ }^{2}$ Rehabilitation Medicine Department, Changchun University of Chinese Medicine, Changchun, China

${ }^{3}$ Acupuncture Department, Henan University of Traditional Chinese Medicine, Zhengzhou, China

${ }^{4}$ TCM Department, Changchun University of Chinese Medicine, Changchun, China ${ }^{5}$ Rehabilitation Medicine Department, Jilin University Third Affiliated Hospital, Changchun, China

${ }^{6}$ Tuina Department, First Affiliated Hospital to Changchun University of Chinese Medicine, Changchun, China

${ }^{7}$ Graduate School, Changchun University of Chinese Medicine, Changchun, China

Contributors QZ and BS conceived and designed the protocol. QZ and YS registered the protocol review in the PROSPERO database and drafted the manuscript. $Y L$ and $J C$ designed the search strategy. QZ and $F C$ drafted the protocol. QZ, GJ, FC, YS, SS, GH, YL, JC, XX, YW, XX and BS contributed to and approved the final manuscript of the protocol review.

Funding This article is supported by the National Key R\&D Program of China which is supported by the Ministry of Science and Technology of the Peoples' Republic of China (grant number 2018YFC1706000).

\section{Competing interests None declared.}

Patient and public involvement Patients and/or the public were not involved in the design, or conduct, or reporting, or dissemination plans of this research.

Patient consent for publication Not required.

Provenance and peer review Not commissioned; externally peer reviewed.

Open access This is an open access article distributed in accordance with the Creative Commons Attribution Non Commercial (CC BY-NC 4.0) license, which permits others to distribute, remix, adapt, build upon this work non-commercially, and license their derivative works on different terms, provided the original work is properly cited, appropriate credit is given, any changes made indicated, and the use is non-commercial. See: http://creativecommons.org/licenses/by-nc/4.0/.

ORCID iD

Qiongshuai Zhang http://orcid.org/0000-0003-0594-1654

\section{REFERENCES}

1 Zhou M, Wang H, Zeng X, et al. Mortality, morbidity, and risk factors in China and its provinces, 1990-2017: a systematic analysis for the global burden of disease study 2017. Lancet 2019;394:1145-58.

2 Strong K, Mathers C, Bonita R. Preventing stroke: saving lives around the world. Lancet Neurol 2007;6:182-7.

3 Raluy-Callado M, Cox A, MacLachlan S, et al. A retrospective study to assess resource utilization and costs in patients with post-stroke spasticity in the United Kingdom. Curr Med Res Opin 2018;34:1317-24.

4 Summers D, Leonard A, Wentworth D, et al. Comprehensive overview of nursing and interdisciplinary care of the acute ischemic stroke patient: a scientific statement from the American heart association. Stroke 2009;40:2911-44.

5 Mahmood A, Veluswamy SK, Hombali A, et al. Effect of transcutaneous electrical nerve stimulation on spasticity in adults with stroke: a systematic review and meta-analysis. Arch Phys Med Rehabil 2019;100:751-68.

6 Watkins CL, Leathley MJ, Gregson JM, et al. Prevalence of spasticity post stroke. Clin Rehabil 2002;16:515-22.

7 Wissel J, Manack A, Brainin M. Toward an epidemiology of poststroke spasticity. Neurology 2013;80:S13-19.

8 Lundström E, Smits A, Borg J, et al. Four-fold increase in direct costs of stroke survivors with spasticity compared with stroke survivors without spasticity: the first year after the event. Stroke 2010;41:319-24. 
9 Zorowitz RD, Gillard PJ, Brainin M. Poststroke spasticity: sequelae and burden on stroke survivors and caregivers. Neurology 2013;80:S45-52.

10 Shiner CT, Vratsistas-Curto A, Bramah V, et al. Prevalence of upperlimb spasticity and its impact on care among nursing home residents with prior stroke. Disabil Rehabil 2020;42:1-8.

11 Khanittanuphong P, Leelasamran W. Assessing caregiver burden and relationship between caregiver burden and basic activities of daily living in stroke patients with spasticity. J Med Assoc Thai 2016;99:926-32.

12 Martin A, Abogunrin S, Kurth $\mathrm{H}$, et al. Epidemiological, humanistic, and economic burden of illness of lower limb spasticity in adults: a systematic review. Neuropsychiatr Dis Treat 2014;10:111-22.

13 Demetrios M, Khan F, Turner-Stokes L, et al. Multidisciplinary rehabilitation following botulinum toxin and other focal intramuscular treatment for post-stroke spasticity. Cochrane Database Syst Rev 2013;6:CD009689.

14 Esquenazi A. The human and economic burden of poststroke spasticity and muscle overactivity. J Clin Outcome Manag 2011;18:607-14.

15 Guo T. Comparison of the therapeutic effects of three kinds of oral antispasmodic drugs on limb spasm after comparison of the therapeutic effects of three kinds of oral antispasmodic drugs on limb spasm after stroke. Chinese $J$ Rehab Theory Pract 2012;18:453-5.

16 Lindsay P, Furie KL, Davis SM, et al. World stroke organization global stroke services guidelines and action plan. Int J Stroke 2014;9:4-13.

17 Foundation NS. Clinical guidelines for stroke management 2010, 2010: 1-167.

18 Wang C, Zhang X, Wang D, et al. Tuina for functional constipation: a protocol for the systematic review of randomized clinical trials. Medicine 2019;98:e14775.

19 Fan Z, Tian Q, Guo R, et al. Tuina for low back pain: protocol for a systematic review and meta-analysis. Medicine 2018;97:e11979.
20 Yang A, HM W, Tang JL, et al. Acupuncture for stroke rehabilitation. Cochrane Database Syst Rev 2016;2016:CD004131.

21 Moher D, Shamseer L, Clarke M, et al. Preferred reporting items for systematic review and meta-analysis protocols (PRISMA-P) 2015 statement. Syst Rev 2015;4:1.

22 Hatano S. Experience from a multicentre stroke register: a preliminary report. Bull World Health Organ 1976;54:541-53.

23 Meseguer-Henarejos A-B, Sánchez-Meca J, López-Pina J-A, et al. Inter- and intra-rater reliability of the modified Ashworth scale: a systematic review and meta-analysis. Eur J Phys Rehabil Med 2018;54:576-90.

24 Gregson JM, Leathley M, Moore AP, et al. Reliability of the tone assessment scale and the modified Ashworth scale as clinical tools for assessing poststroke spasticity. Arch Phys Med Rehabil 1999;80:1013-6.

25 Chen C-L, Chen C-Y, Chen H-C, et al. Responsiveness and minimal clinically important difference of modified Ashworth scale in patients with stroke. Eur J Phys Rehabil Med 2019;55:754-60.

26 Guiu-Tula FX, Cabanas-Valdés R, Sitjà-Rabert M, et al. The efficacy of the proprioceptive neuromuscular facilitation (PNF) approach in stroke rehabilitation to improve basic activities of daily living and quality of life: a systematic review and meta-analysis protocol. BMJ Open 2017;7:e016739.

27 Lindsay C, Kouzouna A, Simcox C, et al. Pharmacological interventions other than botulinum toxin for spasticity after stroke. Cochrane Database Syst Rev 2016;10:CD010362.

28 Aamann L, Dam G, Rinnov AR, et al. Physical exercise for people with cirrhosis. Cochrane Database Syst Rev 2018;12:CD012678.

29 Guyatt GH, Oxman AD, Vist GE, et al. GRADE: an emerging consensus on rating quality of evidence and strength of recommendations. BMJ 2008;336:924-6.

30 jun W, yan L. Literature review of modern Chinese massage disease spectrum. Inform Tradit Chinese Med 2011;28:151-3. 